improved conditions the experiments referred to in our letter, we have been able to show with both erythrosine, an acid dye, and with polymethine (cyanine) basic dyes, that the values for the photochemical equivalent cluster closely around unity. That is to say, for each quantum of light absorbed per dye molecule, one atom of silver is produced, in any event in the initial high-efficiency region of the adsorption ${ }^{1}$. This result shows that Scheibe's hypothesis is unnecessary, as an explanation of the energetics, and, for the region studied, is incorrect.

Research Laboratories,

Eastman Kodak Company, Rochester, N.Y.

Aug. 8.

I NATURE, 140, 1096 (Dec. 25, 1937).

'Scheibe, G., Naturwiss., 25, 795 (1937).

\section{Diffusion in Non-Ideal Media}

IN $1934^{1}$, it was suggested that the high temperature coefficients frequently found for diffusion processes in biological systems were a simple and necessary concomitant of the high potential energy barriers which prevent free diffusion in such systems. It has now been found that the underlying theory can be put into a useful quantitative form.

In an ideal diffusion medium, diffusion is a continuous process, and its rate is defined by the classical equation

$$
D m^{\frac{1}{2}}=\text { constant, }
$$

where $D$ is the diffusion constant and $m$ is the mass of the diffusing molecules. In real liquids and solids, diffusion is retarded by potential energy barriers; hence of the total number of molecules in a system, only those are free to diffuse which have kinetic energy sufficient to permit passage through the potential energy barriers. Hence diffusion alternates with periods of vibration about a mean position.

If the simplest possible assumptions are made about such a system, that is, that the potential barriers surrounding the molecules concerned are uniform and do not vary with time, and that all molecules having sufficient energy to diffuse through the potential energy barriers have in fact an equal chance of doing so, then it is easy to prove the following relationships, (2) for molecules diffusing in a concentration gradient, (3) for ions diffusing in a potential gradient :

$$
\begin{aligned}
D m^{\frac{2}{2}} Q_{10}^{n} & =A \\
l Q_{10}^{n} & =B, \quad . \quad . \quad . \quad . \quad .
\end{aligned}
$$

where $n=(T+10) / 10, T$ is the absolute temperature, $l$ is the mobility of the ions, and $Q_{10}$ is the ratio of the rates of the diffusion process concerned at the temperatures $T$ and $(T+10) . A$ and $B$ are constants independent of the diffusing species, but varying with temperature and with the nature of the diffusion medium. These equations are reasonably satisfactory over a wide range of conditions.

Another method of verifying equation (3) is to use it for the calculation of liquid junction potentials. If the equation is correct, $B / Q_{10}^{n}$ can be substituted for $l$. If this is carried out in Henderson's procedure, it is found that liquid junction potentials can be calculated if the activities and the $Q_{10}$ 's of the mobilities of the ions are known. The difference between the calculated and the observed potentials is of the order of 0.2 millivolts; this is considerably better agreement than is obtained by calculating directly from the mobilities. There is no theoretical reason why the calculation based on $Q_{10}$ should be more accurate than that based on mobility ; the advantage probably arises from the fact that in the measurement of $Q_{10}$ systematic errors tend to cancel out.

Equations (2) and (3) can be applied to biological systems, for the elucidation of membrane structure. If a membrane behaves as a homogeneous structure towards penetrating molecules, equations similar to (2) and (3) hold. It is found that red blood cell membranes are far from homogeneous, whereas Arbacia egg membranes are probably homogeneous.

Experiments are now being made to obtain similar information for nerve and muscle cells.

J. F. DANIELLI.

Department of Physiology and Biochemistry, University College, London, W.C.1. July 26.

${ }^{1}$ Danielli and Davson, J. Cell. Comp. Physiol., 5, 502 (1934).

\section{Isolation of Progesterone and Allopregnanolone from the Adrenal}

THE presence in the adrenal gland of material possessing progestational activity was reported by Engelhart ${ }^{1}$, who obtained lipoid extracts which produced œstrous and progestational effects in the uterus of the immature rabbit. Callow and Parkes ${ }^{2}$ confirmed these findings, and showed that a fractionation of the active materials was possible by the method of Allen and Meyer ${ }^{3}$. They suggested, in view of the structure of the substances already isolated from the adrenal cortex, that the material responsible for the progestational activity might be progesterone or a closely allied compound.

Using pentane-soluble material kindly supplied by N. V. Organon Oss, prepared by extracting ox adrenals by the modified method of Swingle and Pfiffner ${ }^{4}$, followed by distribution of the crude extract between pentane and 30 per cent alcohol, we have each succeeded independently in isolating progesterone (m.p. $121^{\circ}$ ) and allopregnanol(3)-one(20) (m.p. 198-200 cor.). The latter, which is without obvious biological activity, occurred in the greater amounts. Bioassays carried on throughout the work by one of us (D. B.), with the kind co-operation of Dr. A. S. Parkes, suggest that much, if not all, the activity can be accounted for by the progesterone content.

Experimental details of the methods used by each of us for the isolation of these compounds will be published independently in full detail shortly.

$$
\text { D. Beall. }
$$

(Beit Memorial Fellow.)

British Postgraduate Medical School, London.

Chemisches Institut,

T. Retchstein.

Eidg. Tech. Hochschule, Zürich.

Aug. 3.

1 Engelhart, E., Klin. Wochr., 9, 2114 (1930)

Callow, R. K., Parkes, A. S., J. Physiol., 87, 28 P (1936).

${ }^{3}$ Allen, W. M., Meyer, R. K., Amer. J. Physiol., 106, 55 (1933).

" Pfiffner, J. J., Vars, H. M., Taylor, A. R., J. Biol. Chem., 106, 625 (1934). 